\title{
Retrospectiva y reflexiones aproximadas acerca de la degradación del medio ambiente'
}

\section{Approximate retrospective and reflections on environmental degradation}

\author{
Nelson Álvarez Marín
}

FORMA DE CITACIÓN

Álvarez, N. (2013), Retrospectiva y reflexiones aproximadas acerca de la degradación del medio ambiente. Revista dimensión Empresarial, vol. 11, núm. 2, pp. 26-35.

\section{RESUMEN}

Este documento tiene como propósito describir las posturas teóricas y las divergencias surgidas entre los economistas neoclásicos y de aquellos economistas que hallaron en la física un soporte mucho más consistente para su teorización, al trasladar a su campo de estudios la segunda ley de la termodinámica - la ley de la entropía, para apoyar sus razonamientos en cuanto a la disipación de la energía y la materia en el medioambiente, con el fin de demostrar que los economistas convencionales al aplicar el modelo circular producción-mercado-consumo y la sustitución de capital, consideraron dentro de su modelo el agotamiento de los recursos naturales. De esta manera han sido coparticipes con la degradación y contaminación medioambientales. Por estas razones se consideró oportuno hacer notar, desde una mirada sistémica, cómo es posible conciliar a las dos corrientes de pensamiento enfrentadas, al pensar la situación medioambiental como una relación sistema/entorno. Se efectúo para ello, un rastreo a partir de los años 70 sobre la preocupación de las instituciones internacionales y las organizaciones, y de la sociedad civil en general, por la búsqueda de alternativas de solución para afrontar la crisis del medio ambiente contemporáneo; planteándose así, las nociones de sostenibilidad (sustentabilidad) y las corrientes creadas a partir de esta categoría: la sostenibilidad fuerte y la débil. Entendiendo que el fomento de la responsabilidad social y ambiental corporativas trajo consigo dispositivos que se orientan a observar, controlar, costear e intervenir las acciones de las organizaciones al impactar estas el medio ambiente como lo son: la auditoría ambiental y la contabilidad ambiental.

Palabras clave: Medio ambiente, entropía, sistema/entorno, sustentabilidad, auditoría ambiental, contabilidad ambiental.

\section{ABSTRACT}

This document is to describe the theoretical positions and disagreement among neoclassical economists and economists those found in physics much more consistent support for theorizing, to move his field of study to the second law of thermodynamics - the law of entropy, to support their arguments in terms of the dissipation of energy and matter in the environment, in order to show that conventional economists to apply the circular pattern-market production-consumption and capital replacement, considered in their model the depletion of natural resources. In this way have been partakers with environmental degradation and pollution. For these reasons it was considered appropriate to note, from a systemic perspective, how is it possible to reconcile the two opposing schools of thought, thinking about the environmental situation as a relationship system / environment. For it was effected, a crawl from the 70s to the concern of international institutions and organizations, and civil society in general, by the search for alternative solutions to address the contemporary environment crisis, considering notions of sustainability and currents created from this category: the strong and weak sustainability. Understanding that the promotion of corporate social and environmental responsibility brought with devices that are directed to observe, monitor, and intervene afford actions impact these organizations to the environment such as: environmental auditing and environmental accounting.

Keywords: Environment, entropy, system / environment, sustainability, environmental auditing, environmental accounting.

Artículo de reflexión. Fech a de recepción julio 24 de 2013. Fecha de aceptación septiembre 13 de 2013.

Administrador de Empresas, Universidad Externado de Colombia, especialista en Gerencia de Mercadeo, Universidad Central, magister en Relaciones Internacionales, Universidad Javeriana, Docente-investigador Universidad Central, Facultad de Ciencias Administrativas y Contables, Departamento de Contaduría Pública, Bogotá, Colombia. Vinculado al grupo de investigación Mercado, intercambio, cultura y organizaciones, clasificado en COLCIENCIAS, Bogotá, Colombia. Correo electrónico: nalvarezm@ucentral.edu.co 


\section{INTRODUCCIÓN}

El artículo aquí comprendido es el resultado investigativo de una revisión documental llevada a cabo en el año 2012, cuyo propósito principal fue rastrear lo escrito sobre el tema medioambiental y su advenimiento en un pasado cercano. Las pesquisas iníciales se concentran en averiguar sobre la producción académica de distintas corrientes doctrinales las cuales desde sus diversos fundamentos teóricos pretenden esclarecer, comprender y dar posibles soluciones al fenómeno actual de la degradación medioambiental. Algunos de estos enfoques teóricos han sido adoptados por las instituciones públicas y privadas, procurando desarrollar e implementar mecanismos y herramientas de control que armonicen las relaciones de producción-mercado-consumo, mediante un uso más apropiado y eficiente de los recursos naturales atenuando el impacto medioambiental, para proteger la supervivencia de las generaciones actuales, así como de las futuras en el planeta.

La investigación cuyo título original es: Una retrospectiva y reflexiones aproximadas sobre la degradación en el proceso económico, la sustentabilidad y el control del medio ambiente y de la cual se origina el artículo acá contenido, que se relaciona con los intereses trazados desde la línea de investigación titulada: Dinámicas contemporáneas de los mercados y de las relaciones de intercambio, la que ha sido gestada y desarrollada en el grupo avalado por Colciencias y denominado: Mercado, intercambio, cultura $y$ organizaciones ${ }^{3}$, cuyo objetivo dentro de la línea de estudio se dirige a profundizar en el conocimiento de las relaciones de intercambio y de mercado con respecto a la vida social, cultural y económica en la sociedad, así como de los impactos medioambientales derivados de esas relaciones de intercambio.

\section{Problematización y cuestionamientos}

El interés por el estudio de las condiciones medioambientales, afectado por la interacción del hombre y de sus diversos sistemas productivos a través de su historia, ha sido una preocupación de varios estudiosos de diversas disciplinas del conocimiento.

Con todo, no es desconocido hoy en día el resultado de lo sobrevenido al medioambiente global con relación a la Revolución Industrial y de sus comienzos en la segunda mitad del siglo VIII e inicios del XIX, en principio precedida por la Gran Bretaña, para posteriormente extenderse por la Europa Continental y luego a los Estados Unidos, hasta

3 Grupo de investigación anexo a la Facultad de Ciencias Administrativas, Económicas y Contables de la Universidad Central, institución que financia y auspicia la elaboración de este tipo de proyectos de investigación. abarcar en el transcurso del siglo XX diferentes países en el mundo, los cuales han sufrido como resultado de la industrialización las mayores transformaciones socioeconómicas, culturales, tecnológicas y medioambientales de que se tenga conocimiento en toda la historia de la humanidad.

Posterior a la Segunda Guerra Mundial, los procesos acelerados de reconstrucción y recuperación económicos de los países involucrados en la conflagración, y con preeminencia de aquellos implicados directamente en la denominada "guerra fría", se inicia una competencia mundial de gran magnitud, imprimiéndole fuerza y continuidad notable al legado aportado por la "Revolución Industrial". Comprenden ese periodo desarrollos de nuevas tecnologías aeroespaciales, comunicacionales, nucleares, industriales y agroindustriales, entre muchas otras.

Estas economías industrializadas como las emergentes o subdesarrolladas, una vez declinado el modelo socialista de economía planificada en la década de los años 90 , a excepción en la actualidad de algunos países, y con el advenimiento de las políticas neoliberales, las naciones entraron en una competencia por la participación de los mercados globales iniciada por las grandes economías de libre comercio, acelerando de paso el impacto sobre el medioambiente.

Estos acontecimientos alrededor del fenómeno medioambiental conducen a establecer interrogantes acerca de la problemática como:¿Qué le acontecía a las sociedades de la posguerra con la celeridad que presentaba un mundo asediado por el crecimiento demográfico, urbano, industrial, tecnológico y del consumo; así como cuáles sucesos precedían a las tensiones causadas al medio ambiente? ¿Cuáles teorías no ortodoxas se interesaron y preocuparon por los fenómenos de la degradación medioambiental y sus consecuencias para la humanidad en contraposición a los planteamientos económicos ortodoxos? ¿Por qué desde una perspectiva sistémica puede conciliarse la teoría económica clásica y la no ortodoxa en beneficio de un mejor tratamiento de las cuestiones medioambientales? ¿Cómo se observaba la preocupación por esta problemática medioambiental por parte de los estados, gobiernos e instituciones internacionales y de la sociedad en general? ¿Qué compromisos se han adquirido por parte de las diversas entidades y organismos involucrados, además de ser muchos de ellos responsables por la problemática, y qué están haciendo actualmente esas instituciones y empresas para sortear la delicada situación medioambiental?

Interés de la investigación, justificación aspectos metodológicos y objetivos

Esta problemática y los interrogantes con relación al fenómeno medio ambiental conllevan a justificar desde la 
investigación realizada, la necesidad de la comprensión del acontecer y de los distintos sucesos que han propiciado un interés de dimensiones globales durante las últimas cuatro décadas del siglo pasado y en los albores del presente siglo XXI, con relación al fenómeno medioambiental y del futuro común que le depara a las generaciones venideras, en correspondencia con los acontecimientos e intervenciones sobre el medio ambiente de parte de las generaciones actuales.

La metodología adoptada en el proceso de investigación es del tipo revisión documental, en donde este enfoque permite moverse cuidadosamente entre las posibilidades de la construcción de comprensiones económico-socioambientales y la conexión de esta dinámica en torno la acción de realizar una articulación concreta del trabajo en donde se permiten exponer niveles descriptivo, explicativo y comprensivo con relación al devenir de las cuestiones medioambientales.

Los objetivos de la investigación adelantada persiguieron: a) Entender la importancia de las posturas teóricas adoptadas por los economistas ortodoxos y las implicaciones de la aplicación de sus modelos sobre las consecuencias de la degradación ambiental versus la posición adoptada por académicos que ven en los planteamientos mecanicistas de los economistas clásicos una problemática medioambiental, al desconocer el desgaste de los recursos naturales vía la segunda Ley de la Termodinámica, -la entropía; b) Hacer notar desde una mirada sistémica, cómo es posible conciliar a las dos corrientes de pensamiento antes mencionadas al decantar la situación medioambiental como una relación sistema/entorno; c) rastrear a partir de los años 70's, las preocupaciones de las instituciones internacionales, de los estados, de los gobiernos, de las ONG's, de organizaciones empresariales y de la sociedad civil en general por buscar alternativas de solución, mecanismos y herramienta de control para afrontar la crisis de los acontecimientos en los asuntos del medio ambiente contemporáneos; d) Identificar las nociones de sostenibilidad (sustentabilidad) y las corrientes de pensamiento creadas a partir de esta categoría como: la sostenibilidad fuerte y débil, y entender como esta división de la sustentabilidad va a consolidar dos movimientos definidos para deliberar sobre lo concerniente a los asuntos del manejo de las cuestiones del medioambiente: desarrollismo y ambientalismo; y, e) Comprender desde la perspectiva de la responsabilidad social y ambiental corporativas, los mecanismos, herramientas y dispositivos de control desarrollados y orientados a observar, controlar, costear e intervenir las acciones de las organizaciones al impactar estas el medio ambiente en un sistema de economía capitalista, tales como: la auditoría y la contabilidad ambientales.

\section{REFERENTES TEORICO-CONCEPTUALES: LA ECONOMÍA «MECANICISTA» VERSUS LA SEGUNDA LEY DE LA TERMODINÁMICA «LA ENTROPIA» Y UNA MIRADA SISTÉMICA CONCILIATORIA DE LAS DOS POSICIONES}

En este aparte ha de hacerse referencia a los postulados teóricos de los economistas clásicos o mecanicistas versus los planteamientos del economista rumano Nicholas Georgescu-Roegen, con el fin de enriquecer la disertación con relación a los primeros indicios de preocupación por la situación resultante de la contaminación y el agotamiento de los recursos no renovables.

En su libro titulado La Ley de la Entropía y el Proceso Económico, Georgescu-Roegen, en su disertación comenta que la ciencia económica ha sido criticada tal vez como ninguna otra ciencia lo haya sido, y de todas estas amonestaciones una de las principales ha girado alrededor del mito constituido por el homo oeconomicus, ya que el principal motivo de queja es que tal ficción despoja a la conducta humana de toda propensión cultural, lo que equivale a decir que, en su vida económica, el hombre actúa mecánicamente. Esta es la razón por la que se califica habitualmente esa deficiencia de perspectiva mecanicista de la economía moderna (Georgescu-Roegen, 1996, p. 45).

Como economista Georgescu-Roegen difería de las argumentaciones teóricas de corte mecanicista establecidas durante más de cien años por los fundadores de la Escuela Neoclásica. Él entendía que esta obstinada tendencia por pensar a la economía desde los principios de la mecánica, radicaba en el auge que durante la primera mitad del siglo diecinueve obtuvo la ciencia de la mecánica en astronomía, y admite que esa fue una circunstancia atenuante, que motivo a los economistas de la época como William Stanley Jevons a tratar de crear una ciencia económica ajustada al modelo de la mecánica, tal como lo dijera el mismo Jevons una a modo de mecánica de la utilidad y el lucro (Jevons, citado en Georgescu-Roegen, 1975, p. 94).

Lo que no dejaba pasar por alto y reprochaba GeorgescuRoegen, era aprobar que los economistas posteriores a la proscripción del reconocimiento de la mecánica como el modelo universal por parte de la física, continuaran éstos empeñándose en desarrollar el pensamiento económico con base en la opción mecánica de la ciencia. El perpetuar la fidelidad por este dogma mecanicista, reducía la ciencia económica a una mera cinética intemporal enmarcada por unos análisis econométricos que las más de las veces el resultado de esos estudios eclipsan los planteamientos principales. Aduce el profesor que todos los estudios se sujetan a un movimiento pendular en donde la conclusión de un ciclo comercial produce el inicio de otro y así sucesivamente. 
Es por esto que el académico criticaba que la base de la teoría del equilibrio es que, si los hechos alteran el curso de la demanda y de la oferta, el proceso económico vuelve siempre a sus condiciones previas (Georgescu-Roegen, 1975, p. 94), de tal suerte que, por ejemplo, un suceso como un crash bursátil no dejaría huella en una economía, puesto que tal como acontece en la mecánica como norma, todo es reversible. En este orden de ideas, el proceso económico no debe considerarse como un flujo aislado y autónomo entre la producción y el consumo, por el contrario el proceso se ve afectado por una interrelación con el medio circundante: el entorno. Este intercambio que se presenta de manera continua en el proceso económico se torna acumulativo y por ende esas variaciones afectan el medio ambiente para la vida del hombre y de otras especies.

Debido a esto, es que la Primera Ley de la Termodinámica es idéntica a la ley de la conservación de la energía mecánica; luego, en tanto que el trabajo es una de las múltiples formas de energía, esta primera ley de la termodinámica evidencia la ficción del movimiento perdurable de primer orden, es decir que: [...] cualquier proceso puede producirse hacia adelante o hacia atrás, de modo que todo quede otra vez como al principio, sin ninguna huella de lo sucedido, por lo tanto, no se solventa la diferencia entre energía disponible y energía no disponible, por eso: Con esta ley solamente seguimos aún en la mecánica, y no en el campo de los fenómenos reales, entre los que sin duda se encuentra el proceso económico (Georgescu-Roegen, 1975, mayo, p. 97).

De ahí que en la argumentación de Georgescu-Roegen, se declare que el postulado de la primera ley de la termodinámica se equipara al movimiento pendular (o circular) en la física mecánica; por ello, es sólo en la Segunda Ley de la Termodinámica en donde se presenta la oposición irreductible entre la termodinámica y la mecánica, al enunciar esta ley que

[...] la entropía de un sistema cerrado aumenta continua e irrevocablemente hacia un punto máximo, o sea, la energía disponible es transformada continuamente en energía no disponible hasta que desaparece completamente, en otras palabras: Todos los tipos de energía se transforman gradualmente en calor y el calor termina por disiparse hasta un punto en que el hombre ya no puede utilizarla ${ }^{4}$ (Georgescu-Roegen, 1975, mayo, p. 97).

4 Según lo comenta el economista Carpintero, sobre esta argumentación Georgescu-Roeguen gustaba de ejemplificar dicho postulado con perspicacia cuestionándose sí: ¿Sería posible volver a reunir todas las pequeñas partículas de los neumáticos de los coches que por rozamiento con la calzada se dispersan por todo el territorio? ¿Cuánta energía sería necesaria para llevar a cabo esa operación? ¿Tendría sentido económico y energético llevarla a cabo? (1999, julio-agosto, p.134).

\section{UN ENFOQUE SISTÉMICO CONCILIA LA POSTURA DE LA ECONOMÍA NEOCLÁSICA CON LA SEGUNDA LEY DE LA TERMODINÁMICA -LA ENTROPÍA}

Para lograr conciliar los planteamientos de los economistas neoclásicos y los de Georgescu-Roegen desde un enfoque sistémico, ha de comprenderse la existencia en el sistema/ entorno de la preservación de unos límites como condición para la relación y coexistencia del sistema/entorno. Lo anterior determina que cualquier análisis teórico-sistémico precisa para comenzar su estudio del entendimiento de la constitución de la diferencia entre sistema y entorno. En este sentido, se piensa el entorno no como un sistema, sino por lo contrario, el entorno alcanza su "unidad" a través de la relación continua con el sistema. Lo anterior conlleva a imaginar el entorno demarcado por horizontes abiertos y no por límites que se puedan sobrepasar. Esta situación hace pensar en la exclusión de cualquier representación total de dependencia entre sistema/entorno, en tanto que el uno y el otro se acompañan de la complejidad ${ }^{5}$ (Luhmann, 1998). Por lo tanto, es substancial precisar y hacer distinción entre el entorno de un sistema y los sistemas en el entorno de ese sistema y de las relaciones de subordinación entre los sistemas, puesto que: [...] a cada sistema le es dado representarse el complejo de sus relaciones con el entorno como un entramado desconcertante, pero también como una unidad constituida por él mismo, con tal de exigirse una observación selectiva (Luhmann, 1998, p. 41).

En lo correspondiente a esa posibilidad de exigirse una observación selectiva en tanto sistema que se constituye como una unidad en sí misma, ha de comentarse que el dinero -la diferencia según Luhmann, concede como elemento constitutivo y transaccional en sí mismo -medio de pago al sistema económico su condición de unidad (un sistema económico totalmente monetarizado), lo cual

[...] significa que todas las operaciones económicamente relevantes y sólo ellas se refieren al dinero. Su base son los precios, incluso los precios del mismo dinero. El acontecimiento autopoiético ${ }^{6}$ elemental, la última comunicación ya indivisible y de lo que consiste el sistema, es el pago. Pero también las comunicaciones que no son

5 La complejidad designa el grado de sofisticación de la variedad (funciones, jerarquías y estructuras) de un sistema. La complejidad puede medirse como la variedad de un sistema con respecto a otro. La complejidad refleja y promueve el nivel de adaptación del sistema al entorno. Aquí se entiende que la variedad del entorno es siempre superior a la variedad del sistema (De la Reza, 2010, pp. 153-154).

6 La autopoesis representa una consecuencia de la circularidad y complejidad de todo sistema cuyo comportamiento incluye la reproducción de sí mismo. Es enunciada en el ámbito de la neurofisiología y la actividad perceptiva por H. Maturana y F. Varela (De la Reza, 2010, p. 153). Es importante resaltar que es desde la neurofisiología que N. Luhmann migra esta noción de la autopiesis, como uno de los conceptos fundamentales para la construcción de su teoría. 
pago pueden aludir a los pagos, como las decisiones acerca de inversión o de tasas de interés (Luhmann, citado en Gómez, Vargas y Posada, 2007, p. 42).

Luhmann comenta en cuanto a la evolución del sistema de precios, que en un principio estos estaban controlados por el estado en tanto lo que se buscaba era que los precios fueran justos para la sociedad; y, sin embargo, con el desarrollo posterior de la economía de mercado, los precios comunicaban inestabilidades en relación con la oferta y la demanda.

En la figura No. 1 se representa el sistema económico como una unidad cerrada en sí misma y autorreferencial ${ }^{7}$ porque:

Los sistemas no sólo necesitan adaptarse a su entorno, sino también a su propia complejidad. Tienen que afrontar improbabilidades e insuficiencias internas, y desarrollar disposiciones construidas expresamente para reducir conductas divergentes; sólo así, es probable la existencia de estructuras dominantes (Luhmann, 1998, p.54).

De modo que el sistema económico (Ver figura 1) en tanto sistema social se cierra operativamente en lo relativo al mercado y a su entorno que es el natural de donde extrae los recursos (materias primas -baja entropía), los procesa físicamente para luego devolverlos al entorno físico (residuos y desechos -alta entropía).En lo correspondiente al empleo, el sistema económico involucra los agentes económicos empleados en los procesos inherentes al sistema en tanto estén empleados, de lo contrario aquellas personas que no lo estén se encuentran en el entorno social.

En el caso particular del modelo de económico convencional, este presenta un proceso económico circular cerrado y aislado, en donde desde el tratamiento de los modelos matemáticos se hacen reducciones rigurosas que simplifican la realidad distorsionándola, entendiendo que los sistemas sociales y entre ellos el sistema económico gozan de elevados niveles de complejidad.

Es por eso que en el caso del modelo económico tradicional su sistema cerrado y aislado omite la relación con el entorno físico en el cual

La noción de autorreferencia en un sistema cerrado Luhmann la expresa de la siguiente forma: El concepto de autorreferencia designa la unidad constitutiva del sistema consigo mismo: unidad de elementos, de procesos de sistema. "Consigo mismo» quiere decir independiente del ángulo de observación de otros. El concepto no sólo define, sino también incluye la afirmación de un estado de cosas, ya que sostiene que la unidad sólo puede llevarse a cabo mediante una operación relacionante. En consecuencia la unidad tiene que efectuarse, y no está dada de antemano como individuo, como sustancia o como idea de la propia operación (Luhmann, 1998, p. 55).
[...] parte de la idea de un sistema aislado que requiere una dotación inicial de recursos y una vez puesto en marcha se autoalimenta mediante un fenómeno, inentendible, de reproducción y crecimiento del capital, en su expresión monetaria, que se considera inherente a la actividad económica misma (Gómez, Vargas y Posada, 2007, agosto, p. 34).

\section{DECLARACIONES Y PREOCUPACIÓN POR LA DEGRADACIÓN MEDIOAMBIENTAL MUNDIAL}

En Estocolmo (Suecia) entre el 5 y16 de junio de 1972 se celebró la Conferencia Científica de las Naciones Unidas o mejor conocida como la Primera Cumbre para la Tierra, en donde, mediante Declaración adoptada por los miembros, se propuso en el marco de sus principios: la conservación y mejora del medio humano y un plan de acción que contenía recomendaciones para la acción medioambiental internacional. Es en este espacio donde se habla por primera vez del problema del cambio climático, resultado de detritos y contaminantes y señalando de paso a los gobiernos la importancia de considerar los eventos que pudieran alterar y repercutir sobre el clima. En esa Conferencia se creó el organismo de Naciones Unidas que habría de encargarse de los asuntos medioambientales: el Consejo de Administración del Programa de las Naciones Unidas para el Medio Ambiente (PNUMA) (Jackson, 2007, enero).

Para 1987, la Asamblea General de las Naciones Unidas adopta la Perspectiva Ambiental hasta el año 2000, para que las naciones mediante políticas y programas internacionales logren un desarrollo respetuoso con el medio ambiente. Este acontecimiento da pie para introducir por primera vez el concepto de desarrollo sostenible ${ }^{8}$. Para 1988 adquirieron influencia la problemática del calentamiento global y el deterioro de la capa de ozono en el campo público y políticos internacionales. Esto conllevó la situación a que el PNUMA fundara el Grupo Intergubernamental de Expertos sobre el Cambio Climático (IPCC), este foro se enfoca en el estudio del calentamiento debido al efecto invernadero y los cambios climáticos globales (Jackson, 2007, enero).

Dos conferencias significativas sobre el medio ambiente y el desarrollo en los años 90, resaltaron la necesidad del compromiso global en cuanto al medioambiente y el desarrollo, pero además, se asentó lo relacionado con el

8 En 1983 la ONU constituye la Comisión sobre el Medio Ambiente y el Desarrollo, presidida por el primer ministro de Noruega en ese tiempo Gro Harlem Bruntland, Comisión que luego de investigaciones, análisis, debates, disertaciones y consultas públicas a través del mundo, para 1987 produjeron como resultado de esa exhaustiva tarea la publicación y divulgación del informe titulado Nuestro Futuro Común, conocido también como El Informe Bruntland (Our Common Future, 1987): Ver infra, conceptos y enfoques alrededor de la sostenibilidad medioambiental. 
Figura No. 1. El sistema económico según Luhmann

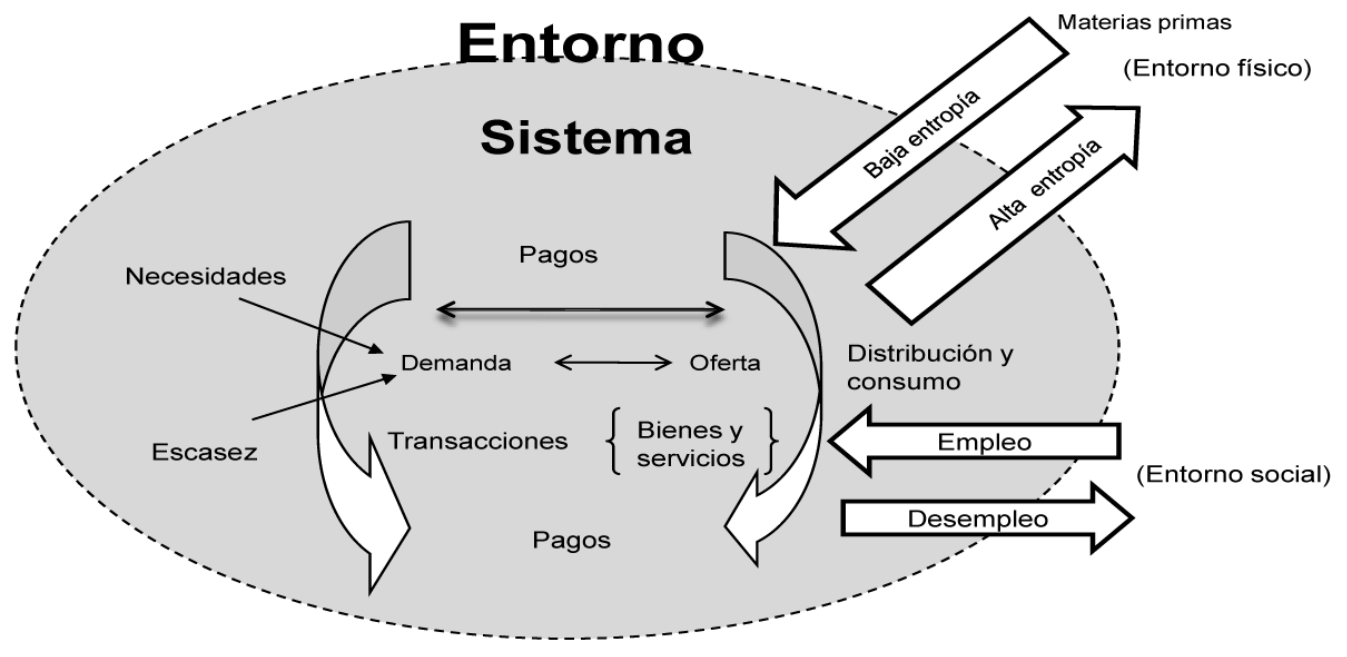

Fuente: Gómez, Vargas y Posada, 2007, p. 42.

desarrollo sustentable en lo concerniente a lo global-local: la Declaración de Rio sobre el Medio Ambiente y el Desarrollo de 1992 y, el Protocolo Kioto de la Convención sobre el Cambio Climático de 1997.

\section{DELIBERACIONESACERCA DE LA SOSTENIBILIDAD DÉBIL Y FUERTE}

Los debates en torno a la sostenibilidad de finales de los años 80 y 90 , aprestaron el desarrollo de dos posiciones de pensamiento importantes frente a las cuestiones medioambientales: la sostenibilidad débil y la sostenibilidad fuerte.

\section{La sostenibilidad débil}

Los economistas neoclásicos Robert Solow, Joseph Eugene Stiglitz, Partha Sarathi Dasgupta y Geoffrey en 1974 publicaron en Review of Economis Studies, sus preocupaciones sobre el crecimiento económico, y el uso de los recursos no renovables en la producción. Estos economistas disertaron sobre cómo incorporar al modelo de crecimiento económico tradicional un nuevo input agregado: los recursos naturales. Este término se relacionaba con los recursos naturales no renovables tenidos en cuenta en la producción. Estos académicos abordaron la situación desde la óptica de la economía convencional y preocupados por la situación se plantearon dos preguntas:

La primera, la más importante y que pertenece a la "economía positiva", consistía en responder a la pregunta de si era posible o no un consumo sostenible de forma indefinida cuando se tiene en cuenta la existencia de recursos no renovables. La segunda cuestión era discutir cuál de las diversas trayectorias de uso de los recursos no renovables era óptima desde el punto de vista social (Martínez y Roca, 2006, p. 375).

Para Solow, la sostenibilidad va a ser el resultado de mantener o conservar el valor del stock de capital, sumado al capital natural, lo que lograría proporcionar un bienestar a las generaciones futuras similar al de las actuales generaciones. El deber de la sostenibilidad se puntualiza en la obligación de conservar un determinado montante de inversión productiva, pues según palabras del economista, el pecado capital no es la extracción minera, sino el consumo de las rentas obtenidas de la minería (Solow, citado en Naredo, 1997, p. 52).

Por lo tanto se precisa lograr la sostenibilidad en términos de la inversión. Esto significa que cuando pueda alcanzarse por medio de la producción y la renta ciertos niveles aceptables, entonces es de esta forma como se obtendrán los excedentes monetarios necesarios y suficientes, para invertirlos en el mejoramiento del medio ambiente. Esta misma fórmula se prescribe para los países menos adelantados los cuales deben anteponer el crecimiento económico a la situación medioambiental hasta tanto no se obtengan los niveles de renta representativos, de tal suerte que habiendo alcanzado esa meta se pueda entonces mejorar la situación medioambiental (Naredo, 1997).

Asimismo, Solow plantea la imposibilidad de prevenir o prohibir el uso o la explotación de los recursos naturales no renovables, ya que, esta actividad de explotación no 
necesariamente implica el total agotamiento y merma de los recursos naturales no renovables. Por ello, este argumento gira alrededor de que los recursos tanto renovales como no renovables son factibles de ser sustituidos por capital. Luego, arguye el académico, esta sustitución puede darse con el uso de nuevos materiales, por ejemplo, utilizar plásticos en lugar de acero, o, menores cantidades de un recurso natural por la misma cantidad de un producto final, como el uso eficiente de la energía. Además, explica el autor que al haber sustituciones de recursos, aquellos recursos que se dejan de utilizar no habrán sido agotados por completo; por eso fue que se sustituyó el uso de carbón vegetal por el de carbón de coque en la industria del hierro, cuando el carbón vegetal se volvió escaso; o como sucedió en otros tiempos con la transición dada desde la caza y la recolección a la agricultura y unida a esta situación la densificación poblacional. En cualquier caso, sin embargo, el capital continúa incrementándose aun cuando los recursos naturales se vuelvan escasos (Solow, citado en Kuhlman y Farrington, 2010).

\section{La sostenibilidad fuerte}

Una respuesta a los postulados propuestos por los economistas convencionales sobre la sostenibilidad, la propone Herman E. Daly, inscrito en la corriente de pensamiento de la sostenibilidad fundamentada en la termodinámica (sostenibilidad fuerte) y quien defendió los argumentos de Georgescu-Roegen en contra de los planteamientos de la sustitución de los recursos naturales por capital y trabajo expuestos por los economistas Solow y Stiglitz.

Daly muestra, en relación con la teoría del crecimiento, que ésta se sustenta en los agregados de la función de producción en donde los recursos naturales no son del todo considerados, puesto que la producción sólo tiene en cuenta las funciones de capital y de trabajo. No obstante, en la realidad la producción requiere de recursos naturales y de materias procedentes de la naturaleza, en donde más que hablar de sustitución se debe hablar de complementariedad ${ }^{9}$. Por eso, sarcásticamente Georgescu-Roegen señalaba que para mantener más adelante un mundo en ausencia de recursos naturales, era algo así como ignorar la diferencia entre este mundo real actual y el Jardín del Edén (Daly, 1997).

Estas posiciones han dado pie a la construcción de lo que se ha denominado la sostenibilidad fuerte. Quienes estudian y se solidarizan con este paradigma, se alejan de los agregados económicos tanto como del concepto de desarrollo,

$9 \quad$ Daly precisa al respecto: Es más, se postula que los elementos y sistemas que componen el "capital natural" se caracterizan más bien por ser complementarios que sustitutivos con respecto al capital producido por el hombre (Naredo, 1997, p. 52). implicándose mejor con el análisis de la sostenibilidad de procesos físicos en un marco espacio-temporal. Es así como el estudio del ámbito espacial tiene una relación con la consecuente disponibilidad de recursos naturales y el tratamiento de los residuos, al observarse este estudio desde la representación de los sistemas físico-sociales en donde la perspectiva temporal concierne, según sean las circunstancias, a probabilidades de llevarse a cabo el proceso físico-social en las mejores condiciones, manteniendo y controlando posibles pérdidas de la calidad tanto internas en el sistema social así como del medioambiente (Naredo, 1997).

Sin embargo, la sostenibilidad ha venido establecido un puente para zanjar la brecha existente entre los seguidores del paradigma desarrollista y los del ambientalista. Por ello, tal como lo argüía el economista Solow, su proposición de controlar los agregados económicos no se encuentra en pugna con, sino de soportarse en conocer mejor el medio ambiente y la manera como los procesos económicos deben estar al tanto e interactuar con el medio ambiente físico, para lograr conciliar la insularidad presentada con la categoría de valor-entropía de la que han venido ocupándose los economista estudiosos de la composición termodinámica medioambiental (Solow, 1986, marzo).

No obstante los seguidores de la economía de la Naturaleza, constituidos por la ecología, y también incorporados en la sostenibilidad fuerte, entienden que las valoraciones económicas y de contabilidad crematísticas no son suficientes para evaluar en su totalidad el impacto sobre el medio ambiente, y por ello, la reflexión ecologista ha hecho un llamado a las sociedades para garantizar la distribución adecuada de energía y de recursos que propendan por mantener la biodiversidad, proporcionando un tratamiento adecuado a los residuos evitando así las externalidades negativas de la contaminación (Gómez, Vargas y Posada, 2007).

Los planteamientos hasta ahora expuestos sobre la sostenibilidad y observados desde diferentes paradigmas, los cuales conllevan a reflexiones en el campo de la economía neoclásica o en el campo de la física o de la ecología como se ha descrito, vienen propiciando el establecimiento de políticas ambientales y de mecanismos de control para tratar los asuntos del medio ambiente.

\section{LA CONTABILIDAD Y LA AUDITORÍA AMBIENTALES COMOMECANISMOSDECONTROLMEDIOAMBIENTALES}

La preocupación suscitada por las cuestiones ambientales ha conducido a las instituciones nacionales e internacionales a establecer políticas y reglas orientadas hacia las organizaciones empresariales, para ejercer un control y conminar a restituir los daños infringidos al medioambiente 0 , contrariamente, a compensar aquellas empresas respetuosas 
de él. Es por esto que en los últimos años ha comenzado a tomar fuerza la auditoría y la contabilidad ambientales.

\section{La Auditoría Ambiental}

En principio es válido sugerir sin lugar a contradicciones que históricamente se ha demostrado que la actividad de auditar existe en ausencia de una legislación segura por parte del aparato regulatorio (Mills, 1990), y por lo tanto, si se habla de leyes ambientales, es en el último cuarto del siglo veinte en donde asalta la preocupación por las cuestiones ambientales y comienza a darse un cambio y desarrollo importantes en el establecimiento de normas para regir las actividades de negocios y de su relación con las afectaciones ecológicas.

En consecuencia es pertinente ilustrar con algunas definiciones el propósito de la auditoría ambiental. Para Carlos Uñi Ugas, la auditoría ambiental se define como:

[...] el proceso de investigación realizado por un auditor independiente, dirigido a determinar el grado de eficiencia empresarial, con relación al grado de satisfacción experimentado por la comunidad y su hábitat, señalando en un informe de auditoría a los agentes degradantes del medio ambiente y de la magnitud de la degradación del medio ambiente (Uñi, citado en Palehn y Frondi de García 2004, p. 200).

Otra definición de la auditoría ambiental la propone la Confederation of British Industry (CBI) en 1990, la cual se soporta a su vez en la definición propuesta por The International Chamber of Commerce (ICC) del año 1989 y dice sobre la auditoría ambiental que es

[...] el examen sistemático de las interacciones entre cualquier operación de negocios y su medio circulante. Esto incluye todas las emisiones hacia el aire, tierra y agua; limitaciones legales; los efectos sobre la comunidad de vecinos, paisaje y ecología; y la percepción que el público tiene respecto de la operación de la compañía en el área local... la auditoría ambiental no se detiene en el cumplimiento de la legislación. No es un ejercicio de relaciones públicas para lavarse en verde... Es más bien una aproximación estratégica a las actividades de la organización (Gray, Bebbington y Walters, 2003, p. 83).

Tal como han estado evolucionadas últimamente las cuestiones ambientales a nivel mundial, las empresas tienen evidencias bastante fuertes donde se sugiere preferiblemente mantener una reputación favorable en los temas relacionados con el medio ambiente, en tanto que una publicidad adversa por parte de los medios en este campo desfavorece la posición comercial de la organización en el mercado. Esta manifiesta situación actual referente a los temas ambientales, ha llevado a varias empresas a optar por las instancias éticas a fin de evitar la mala publicidad y propaganda, y de la misma manera, a tratar de soslayar lides judiciales de alto costo debido a incurrir en el desacato de las normas ambientales; además de prevenir las manifestaciones negativas de los activistas y la pérdida de valor de marca afectando a los inversionistas (Watson y MacKay, 2003). De ahí, por lo tanto, la fuerza y significación adquiridas de parte de las empresas para implementar en su gestión administrativa la auditoría ambiental, circunstancia que se viene propiciando dentro del marco de la responsabilidad social corporativa en los últimos tiempos.

\section{La Contabilidad Ambiental}

Las tensiones y preocupaciones existentes en la sociedad, las organizaciones y el medioambiente por la degradación y merma de los recursos naturales, ha conducido a diferentes instituciones internacionales así como a estados y gobiernos a ir estableciendo paulatinamente políticas, legislaciones, mecanismos y herramientas de control encaminados a corregir y reducirlos impactos infringidos al medioambiente. Para ello se hace necesario contar con un sistema contable potencialmente capaz de suministrar información no sólo relacionada con el patrimonio del ente empresarial, sino el de ir más lejos, al tener la capacidad de informar sobre las actividades de la organización en donde se dé cuenta de su relación con el fenómeno social y con la problemática medioambiental.

La contabilidad ambiental es considerada como uno de los componentes que comprende la contabilidad social. Para acercarse al objeto de conocer la función de la contabilidad ambiental primero se precisa definir lo que se entiende por "responsabilidad social empresarial" (RSE) desde la perspectiva empresarial. Dice al respecto Enrique Herrscher que:

[...] la responsabilidad social, va desde la cooperación de la empresa con la comunidad, especialmente la más inmediata a su zona de influencia, hasta el impacto que tiene su actividad sobre la economía y desarrollo del país. Desde sus responsabilidades en cuanto a la ecología y medio ambiente, hasta -en última instancialos efectos económicos y sociales de sus proyectos e inversión y desinversión (Herrscher, citado en Pahlen y Fronti de García, 2004, p. 169).

Siguiendo a los autores Pahlen y Fronti de García (2004), ellos plantean que varios tratadistas del campo contable involucran a la contabilidad ambiental como uno de los componentes de la contabilidad social, los otros son a saber: la contabilidad de recursos humanos y la referida a la información de carácter ético; pero especialmente Fronti de García indica que debido al nivel de importancia adquirido por los asuntos ambientales en los últimos años, 
esta situación ha llevado a los especialistas a prescribir que la contabilidad ambiental deba de tratarse como un componente con identidad propia.

En esta disposición puede decirse que la sociedad en el transcurso de las últimas décadas ha venido demandando de las empresas la anexión en su gestión en cuanto al objetivo de preservar el entorno social y el medio ambiente en donde desarrollan sus actividades y operaciones. Es por esto que las empresas requieren integrar a sus procesos de gestión factores o variables medioambientales tendientes a generar o crear en su seno políticas y sistemas de gestión medioambientales (Pahlen y Fronti de García, 2004).

Es pertinente sellar con la descripción de algunos conceptos y la forma de clasificación de los costes medioambientales. Consiguientemente, ha de decirse que el concepto de coste está [...] vinculado al sacrificio de recursos que es necesario realizar para poder elaborar un producto de cara a incrementar el valor añadido del mismo, puesto que de lo contrario constituye un despilfarro desde el punto de vista económico (Asociación Española de Contabilidad y Administración de Empresas, 1999, p.43). En consecuencia cuando esos sacrificios se llevan a cabo y afectan la calidad de vida de los seres humanos y de los recursos naturales, se está hablando por lo tanto de los costes medioambientales, los cuales se producen pormenorizadamente cuando se considera el ámbito medioambiental en la Contabilidad de Gestión. De ahí que el objetivo tendiente a la preservación del medio ambiente por parte de las organizaciones, conduce de entrada a las empresas a involucrar una serie de objetivos mínimos en su gestión de control medioambiental, son estos a saber:

- $\quad$ Aprovisionamientos: recursos mínimos y uso extensivo de materiales reciclados y renovables.

- Procesos productivos e inversiones en inmovilizado: mínimo consumo de agua y energía, mínima emisión atmosférica, mínima cantidad de residuos y vertidos.

- Características del output: mínimos envases y embalajes, reciclaje y reutilización de los mismos (Asociación Española de Contabilidad y Administración de Empresas, 1999, p. 37).

\section{A MANERA DE REFLEXION FINAL}

La problemática medioambiental, como se ha descrito es producto de la permanente incursión desmedida y de la acción por parte del hombre sobre la Naturaleza, como en el caso particular del exceso de la explotación indiscriminada y la merma incesante de los recursos no renovables.

Estos hechos han conllevado a una preocupante crisis mundial y con mayor denotación a partir de la Segunda Guerra Mundial como resultado de la contaminación y de- gradación del medio ambiente derivados de una incesante industrialización y del consumo masivo. Pero a pesar de lo grave e inquietante de esta situación ambiental global, lo significativo, es el haberse permitido disparar las alarmas sobre lo delicado de la problemática medioambiental en diversas instancias de la sociedad mundial.

Es así como varios teóricos de diferentes campos del conocimiento científico han contemplado la necesidad de manifestar sus puntos de vista en torno a la problemática ambiental, contribuyendo de este modo para que instituciones internacionales, estados, gobiernos, organizaciones empresariales y la sociedad civil, comenzasen a preocuparse e interesarse por los fenómenos ambientales, mirando y explorando soluciones desde lo político, lo legal, lo económico, lo tecnológico y lo social y empresarial; y, a su vez, también de ir identificando mecanismos para auditar, contabilizar y registrar los impactos e igualmente de posibilitar la recuperación, restablecimiento y conservación medioambientales con miras a mantener unas condiciones mucho más amigables entre las instituciones, las organizaciones y el medioambiente, propendiendo mediante estas determinaciones por procurar una convivencia digna entre nosotros los seres humanos y el medioambiente, para asegurar a esta generación y las futuras, así como de las diversas especies que habitan el planeta un medioambiente mejor preservado.

\section{REFERENCIAS}

Asociación Española de Contabilidad y Administración de Empresas, (1999), Contabilidad de Gestión Medioambiental, Segunda edición, Madrid, Principios de Contabilidad de Gestión, Documento núm. 13.

Carpintero, O. (1999, julio-agosto), Economía y ciencias de la naturaleza: algunas consideraciones sobre el legado de Nicholas Georgescu-Roegen, Tribuna Económica, [en línea], disponible en: http://www.google.com.co/url?sa=t\&rct=j\&q= \&esrc $=$ s\&source $=$ web\&cd $=22 \& v e d=0 C C o Q F j A B O B Q \& u r \mid$ =http $\% 3 \mathrm{~A} \% 2 \mathrm{~F} \% 2 \mathrm{Fwww}$.mdpi.com\%2F2071-1050\%2F2\% 2F11\%2F3436\%2Fpdf\&ei=gzRSUMDJJYnq8wTkjoGgDw\& usg=AFQjCNEeExuFi9ylQKPyA3BtFRM8-yfITw.

Daly, H. E. (1997), Forum Georgescu-Roegen versus Solow/ Stiglitz, en revista Ecological Economics [en línea], disponible en: http://www.eco.unrc.edu.ar/wp-content/uploads/2010/09/ roegen-vs-solow-stiglitz-DALY-1.pdf.

De la Reza, G. (2010), Sistemas complejos: Perspectivas de una teoría general, Barcelona, Anthropos EditorialUniversidad Autónoma Metropolitana.

Georgescu-Roegen, N. (1996), La ley de la entropía y el proceso económico, Madrid, Fundación Argentaria - Visor Distribuciones. 
Georgescu-Roegen, N. (1975, mayo), Energía y mitos económicos, ICE revista de economía [en línea], disponible en:http://www.cima.org.es/archivos/Areas/ciencias_sociales/8 humanidades.pdf.

Gómez L. J., Vargas, E. y Posada, L. G. (2007), Economía ecológica bases fundamentales, Bogotá, IDEA - Universidad Nacional de Colombia.

Gray, R. Bebbington, J. Y Walters, D.(2003), Contabilidad y Auditoría Ambiental, Bogotá, D. C., ECOE Ediciones.

Jackson, P. (2007, 6 de enero), De Estocolmo a Kyoto: Breve historia del cambio climático, Crónica ONU, Naciones Unidas [en línea], disponible en: http://www.un.org/wcm/ content/site/chronicle/lang/es/home/archive/issues2007/ greenourworld/pid/21620.

Jevons, W. S.(2012), Encyclopædia Britannica, Ultimate Reference Site. Chicago: Encyclopædia Britannica.

Kuhlman, T. Y Farrington, J. (2010), What's Sustainability? en: Sustainability Review [en línea], disponible en: http:// ddd.uab.cat/pub/worpap/2006/hdl 2072 1959/48901.pdf.

Luhmann, N. (1998), Sistemas Sociales: Lineamientos para una teoría general, Anthropos, México: Universidad Iberoamericana, Santafé de Bogotá, CEJA: Pontificia Universidad Javeriana.

Martínez, J. Y Roca, J. (2006), Economía ecológica y política ambiental, Segunda edición, México, Fondo de Cultura económica.

Mills, P. A. (1990), Agency, Auditing and the Unregulated Environment: SomeFurtherHistoricalEvidence, Accounting,
Auditing \& Accountability Journal [en línea], vol. 3 núm. 1, disponible en: http://dx.doi.org/10.1108/09513579010003575.

Naredo, J. M. (1997), Sobre el origen, el uso y el contenido del término sostenible, [en línea], disponible en:http:// www.laopiniondelanzarote.com/cuadernos/pdfs/numero03/ sobreelorigeneluso.pdf.

Our Common Future (1987): Report of the World Commission on Environment and Development, United Nations [en línea], disponible en: http://conspect.nl/pdf/Our_Common_FutureBrundtland Report 1987.pdf

Palehn, R. J. M. Y Frondi de García, L. (2004), Contabilidad Social y Ambiental, Buenos Aires, Ediciones Macchi.

Posada, L. G. (1999, noviembre), Nicholas GeorgescuRoegen, Ensayos de Economía [en línea], pp. 194-168, disponible en: http://www.revistas.unal.edu.co/index.php/ ede/article/viewFile/23894/24565.

Solow, R. M. (1986, marzo), On the Intergenerational Allocation of Natural Resources, The Scandinavian Journal of Economics [en línea], vol. 88, núm. 1, disponibleen:http:// links.jstor.org/sici?sici $=03470520 \% 28198603 \% 2988 \% 3 \mathrm{~A} 1$ \%3C141\%3AOTIAON\%3E2.0.CO\%3B2-T

Stiglitz, J. E. (2012), Encyclopædia Britannica, Ultimate Reference Suite. Chicago: Encyclopædia Britannica.

Watson, M. Y MacKay, J. (2003), Auditing for the environment, Managerial Auditing Journal [en línea], vol. 18, núm. 8 pp. 625 - 630, disponible en: http://dx.doi. org/10.1108/02686900310495115. 